\title{
Kinematic and Three-Dimensional Dynamic Modeling of a Biped Robot
}

\author{
E. Hashemi, A. Khajepour, Dept. of Mechanical and Mechatonics Eng., University of Waterloo
}

\begin{abstract}
This article focuses on inverse kinematic formulation and dynamic modeling of the Nao biped robot's lower body, accompanied by verification with the joints' angles as experimental data. Dynamic modeling in two different planes is discussed and joint angles for the given positions, nominal conditions, and trajectory computations are simulated and graphically illustrated. A new approach for development of the inverse dynamics on the aforementioned robot's lower body is proposed in this paper, analytically studied, and compared with MSC Adams for two various scenarios of fixed supporting leg and ground contact implementation.
\end{abstract}

Keywords: Biped robots, inverse kinematics, inverse dynamics, dynamic modeling, trajectory approximation

\section{INTRODUCTION}

Extensive studies have been conducted on various control methods of bipedal walking and stability. Motion study of bipedal robots [1] necessitates correct solutions of the forward and inverse kinematics with optimized and fast closed form computations [2-5] to follow generated trajectories. The kinematic model is also essential to propose a reliable and optimal control method to preserve stability and perform walking tasks as discussed in [6-8], in which the desired zero moment point (ZMP) positions, together with foot pattern generators, provides the desired input for walking. Moreover, biped robot gait and trajectory planning must satisfy several requirements and constraints of bipedal locomotion with diverse degrees of freedom (DOF) from which stability and position of the zero moment point are the most important ones [6,9]. Biped robot's Kinematic-Based Walking Engine uses ZMP criterion to generate joint angles and tries to track the desired ZMP position and keep it, as much as possible, inside the supporting foot contact area [10]. The main core of this type of walking engine contains a three-dimensional linear inverted pendulum module, 3D-LIPM, which employs optimal control of a discrete-time model as a realtime pattern generator for the robot's center of mass. Its inputs are the desired ZMP pulses together with foot pattern generator $[8,10]$. On the other hand, dynamic model is also essential to address the dynamic gait, torque control approaches, and actuators' sizing for the biped robot, but it is complex in general. Thus, a simplified dynamic modeling methodology seems promising.

This paper has different sections to cover: general descriptions of the joint coordinates, closed form representations of forward and inverse kinematics (according to the specified trajectory during a straight walk), and the dynamic model. Finally, the simulation results of joint torques angles will be verified with the MSC Adams outcomes. Geometrical aspects of the selected platform, dedicated coordinates, employed foot trajectories, derivation of the forward and inverse kinematic equations, and a control strategy for the Nao biped robot are provided in section II. The third section deals with formulations of three-dimensional (3D) dynamic model, modeling in two separate planes, and modeling in the MSC Adams multi-body dynamics package. Section IV contains the simulation and experimental results of the joint torques, joint angles, and ground forces for two main case studies (namely: fixed supporting leg and ground contact utilization). Finally, section V describes the conclusions and authors' future works. The Appendix section contains some technical data of the robot and the inverse dynamic equation parameters.

\section{Kinematic Modeling AND TRAJECTORY DEFINITION}

This section presents kinematic formulations, suggested DH frame assignment to be employed in the robot walk engine, and a controller based on a 3D-LIPM as the ZMP generator. The investigated model utilizes foot and torso trajectories as inputs to the inverse kinematic equations.

\section{A. Forward and inverse kinematics}

The Nao biped robot (made by Aldebaran Co., in the RoboCup SPL, Standard Platform League), Fig. 1, has 21 DOF, including 6 in each leg. They include: AnkleRoll, AnklePitch, KneePitch, HipPitch, HipRoll, and HipYawPitch, in which the YawPitch joints of hips are physically bounded and driven with one servo motor. The expression in Cartesian coordinates is done by transforming joint space to Cartesian space where the positions of the links' mass centers and joints are calculated from the given rotation of joints.

AnklePitch, KneePitch, and HipPitch joints are employed for movement of the robot in the $\mathrm{x}$ direction of sagittal plane, and thus have larger operational regions. Both AnkleRoll and HipRoll joints are mainly utilized for movement in the $y$ direction of frontal plane. The HipYawPitch joint has a combinational movement both in the sagittal and transverse planes, since it is mounted 45 degrees with respect to the $y$ and $z$ axes. The main function of this joint is to turn the robot for various path-planning requirements on the field. However, this joint also has a small variation during its stable, straight walk. As a result, this joint is neglected in the formulation development of the dynamic model in Section III because of its negligible variations. The open-loop serial chain system is based on Denavit-Hartenberg (DH) formulations provided in [11, 12]. It is constructed with starting point on the intersection of roll and pitch joints axes of the supporting leg and end point on corresponding point on the non-supporting leg. These frames are aligned with the global reference frame $\{W\}$. Furthermore, the position of the global frame is fixed to the initial frame such that the initial frame always contains the global position. 


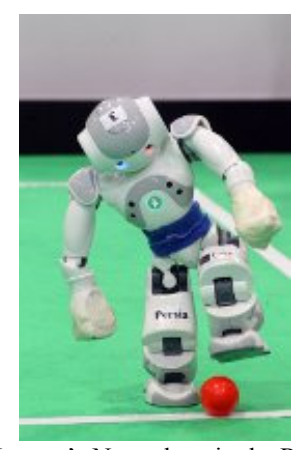

Fig. 1 MRL-SPL team's Nao robots in the RoboCup SPL field

General frame assignments and joint axes of rotations are provided in Fig. 2.

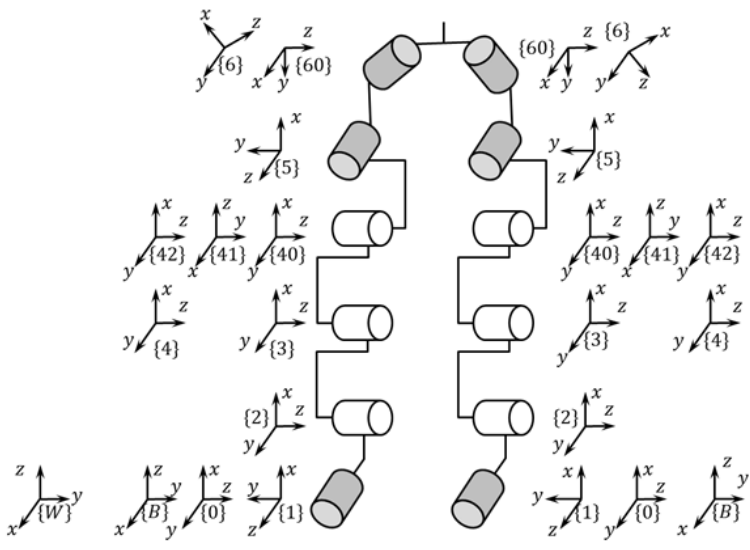

Fig. 2 Nao biped robot legs DH representation

Furthermore, Table A1 and A2 in the Appendix section represent all six joint numbers in each leg, coordinate sequences, and $\mathrm{DH}$ parameters. $\mathrm{DH}$ parameters are dedicated according to the geometrical specifications of two successive moving frames [13]. Joints' rotations are computable with homogeneous transformation $T_{i} \in \mathbb{R}^{4 \times 4}$ :

$$
T_{i}=\left[\begin{array}{cc}
R_{i} & L_{i} \\
0_{1 \times 3} & 1
\end{array}\right]
$$

where $R_{i} \in \mathbb{R}^{3 \times 3}$ is the base rotation matrix of $i^{\text {th }}$ joint about its rotation axes mentioned in Table A1. In addition, $L_{i} \in \mathbb{R}^{3 \times 1}$ is the corresponding position vector of the $i^{\text {th }}$ joint to the $i^{\text {th }}-1$ joint according to joint position values in Table A3, Appendix A. These position data points are extracted from the Nao geometrical specifications. The homogeneous transformation $A_{i}$ between joints $i$ and $i-1$, with multiplication of four rotational and translational matrices can be obtained as:

$$
\begin{aligned}
A_{i} & =R_{z, \theta_{i}} \bar{T}_{z, d_{i}} \bar{T}_{x, a_{i}} R_{x, \alpha_{i}} \\
& =\left[\begin{array}{cccc}
c_{\theta_{i}} & -s_{\theta_{i}} c_{\alpha_{i}} & s_{\theta_{i}} s_{\alpha_{i}} & a_{i} c_{\theta_{i}} \\
s_{\theta_{i}} & c_{\theta_{i}} c_{\alpha_{i}} & -c_{\theta_{i}} s_{\alpha_{i}} & a_{i} s_{\theta_{i}} \\
0 & s_{\alpha_{i}} & c_{\alpha_{i}} & d_{i} \\
0 & 0 & 0 & 1
\end{array}\right] .
\end{aligned}
$$

Matrix $A_{i}$ is a function of only one variable and the three parameters are constant for a link. It should be mentioned that: $\theta_{i}$ for revolute joints and $d_{i}$ for prismatic joints are joint variables according to the $\mathrm{DH}$ formulations assumptions. In (2), $R_{z}$ and $R_{x}$ stand for rotation matrices about the $z$ and $x$ axes, $\bar{T}_{z}$ and $\bar{T}_{x}$ shows translational matrices in both $z$ and $x$ directions, "cos" and "sin" functions are abbreviated with $c$ and $s$ respectively, and other parameters represent DH parameters defined in Table A2. The rotation matrices $R_{x}, R_{y}, R_{z}$ are also conventional ones about the three axes of Cartesian coordinates.

The homogeneous transformation, ${ }_{6}^{0} \mathrm{~T}$, which describes position and orientation of frame $\{6\}$ relative to frame $\{0\}$ is introduced as:

$$
{ }_{6}^{0} T=A_{1} A_{2} A_{3} A_{40} A_{4} A_{5} A_{60} A_{6}=\left[\begin{array}{cc}
{ }_{6}^{0} R & P_{6 O R G}^{0} \\
0 & 1
\end{array}\right] .
$$

In the above homogeneous transformation, the position vector, $P_{6 O R G}^{0}$, expresses the coordinate location of frame $\{6\}$ origin relative to the frame $\{0\}$ origin. Term ${ }_{6}^{0} R$ demonstrates the relative orientation of the two mentioned frames. These homogeneous transformations are utilized to lay out the inverse kinematic model.

The purpose of the inverse kinematic model is to determine joint angles which yield a specific position of the limbs in the Cartesian space. The inverse kinematic model requires expected positions of links, which could be attainable by the torso and foot trajectories. Numerical and closed-form solutions are widely used to solve inverse kinematics problems. Goldenberg et al. [14] presented an iterative numerical solution to transform the desired position of a limb into joint angles by solving the pseudo inverse Jacobian in order to reach accurate results. The main drawback of this method is its computation time for both legs. Therefore, it does not satisfy time response requirements of the Nao biped robot's control loop model with a $500 \mathrm{MHz}$ embedded processor. Closed-form solutions of the kinematic equations of the robot manipulators (DH open chain system) with limited DOF are introduced as an alternative method of solving inverse kinematics equations. This method is utilized to carry out the solution of the robot's kinematic equations using Pieper's solution [11] for developing the transformation and rotation matrices in this paper. Modifications of this method, for the selected Nao platform, are described in this section.

Joint angles $\theta_{1}, \theta_{2}$, and $\theta_{3}$ could be computed by using $P_{6 O R G}^{0}$. Then the last three joint angles of each leg $\theta_{4}, \theta_{5}$, and $\theta_{6}$ are achievable using ${ }_{6}^{0} R$. Calculation of $P_{6 O R G}^{0}$ is vital because it yields the angles $\theta_{1}, \theta_{2}$, and $\theta_{3}$ in the Pieper's solution. Since the origins of the frames $\{4\},\{5\}$, and $\{6\}$ are coincident at a point, then $P_{6 O R G}^{0}$, is equal to $P_{4 O R G}^{0}$, and could be mathematically described as:

$$
\begin{aligned}
P_{6 O R G}^{0}=P_{4 O R G}^{0}= & { }_{3}^{0} T \times P_{4 O R G}^{3} \\
& ={ }_{1}^{0} T \times{ }_{2}^{1} T \times\left[\begin{array}{c}
f_{1}\left(\theta_{3}\right) \\
f_{2}\left(\theta_{3}\right) \\
f_{3}\left(\theta_{3}\right) \\
1
\end{array}\right] .
\end{aligned}
$$

This matrix should be equal to the desired trajectory vector $\left[\begin{array}{llll}x & y & z & 1\end{array}\right]^{T}$ and one may write:

$$
P_{6 O R G}^{0}=\left[\begin{array}{c}
c_{1} g_{1}\left(\theta_{2}, \theta_{3}\right)-s_{1} g_{2}\left(\theta_{2}, \theta_{3}\right) \\
s_{1} g_{1}\left(\theta_{2}, \theta_{3}\right)+c_{1} g_{2}\left(\theta_{2}, \theta_{3}\right) \\
g_{3}\left(\theta_{2}, \theta_{3}\right) \\
1
\end{array}\right]=\left[\begin{array}{c}
x \\
y \\
z \\
1
\end{array}\right],
$$

in which $c_{1}$ and $s_{1}$ stands forcos $\theta_{1}, \sin \theta_{1}$ respectively and $f_{i}, g_{i}$ are functions of DH parameters as [15]:

$f_{1}\left(\theta_{3}\right)=a_{3} c \theta_{3}+d_{4} s \alpha_{3} s \theta_{3}+a_{2}$, 


$$
\begin{gathered}
f_{2}\left(\theta_{3}\right)=a_{3} c \alpha_{2} s \theta_{3}-d_{4}\left(s \alpha_{3} c \alpha_{2} c \theta_{3}+s \alpha_{2} c \alpha_{3}\right) \\
-d_{3} s \alpha_{2}, \\
f_{3}\left(\theta_{3}\right)=a_{3} s \alpha_{2} s \theta_{3}-d_{4}\left(s \alpha_{3} s \alpha_{2} c \theta_{3}-c \alpha_{2} c \alpha_{3}\right) \\
+d_{3} c \alpha_{2} \\
g_{1}\left(\theta_{2}, \theta_{3}\right)=c \theta_{2} f_{1}\left(\theta_{3}\right)-s \theta_{2} f_{2}\left(\theta_{3}\right)+a_{1}, \\
g_{2}\left(\theta_{2}, \theta_{3}\right)=c \alpha_{1}\left[s \theta_{2} f_{1}\left(\theta_{3}\right)+c \theta_{2} f_{2}\left(\theta_{3}\right)\right] \\
-s \alpha_{1} f_{3}\left(\theta_{3}\right)-d_{2} s \alpha_{1} \\
g_{3}\left(\theta_{2}, \theta_{3}\right)=s \alpha_{1}\left[s \theta_{2} f_{1}\left(\theta_{3}\right)+c \theta_{2} f_{2}\left(\theta_{3}\right)\right] \\
+c \alpha_{1} f_{3}\left(\theta_{3}\right)+d_{2} c \alpha_{1} .
\end{gathered}
$$

Therefore, the first three joint angles are attainable with simultaneous solution of:

$$
\begin{aligned}
z=g_{3}\left(\theta_{2}, \theta_{3}\right), & \\
r=x^{2}+y^{2}+z^{2} & =\left[g_{1}\left(\theta_{2}, \theta_{3}\right)\right]^{2}+\left[g_{2}\left(\theta_{2}, \theta_{3}\right)\right]^{2} \\
& +\left[g_{3}\left(\theta_{2}, \theta_{3}\right)\right]^{2} .
\end{aligned}
$$

Parameters, $s \alpha_{1}$ and $c \alpha_{1}$ in $g_{2}$ and $g_{3}$ functions, are required for the solution of (7) and could be extracted from the robot's geometrical specifications in Table A2. Joint angle $\theta_{1}$ is also available after the calculation of $\theta_{2}$ and $\theta_{3}$, and after substituting those results into (5). The closed formulations of the AnkleRoll, AnklePitch, and KneePitch joint angles, with respect to the specified trajectories are provided in the Appendix. The rotation matrix ${ }_{3}^{0} R$, is obtained with determination of $\theta_{1}, \theta_{2}$, and $\theta_{3}$. Using the rotation matrices between the $\{0\},\{3\}$ frames and $\{0\},\{6\}$ frames, one can write the rotation matrix between frames $\{3\}$ and $\{6\}$ as:

$$
{ }_{6}^{3} R=\left({ }_{3}^{0} R\right)^{-1} \times{ }_{6}^{0} R .
$$

The rotation matrix ${ }_{6}^{3} R$ between frames $\{3\}$ and $\{6\}$ includes the remaining three joint angles $\theta_{4}, \theta_{5}$, and $\theta_{6}$. Introducing the frame $\{41\}$ on the hip and parallel to the base frame, as shown in Fig. 2 and Table A2, yields:

$$
{ }_{6}^{41} R=\left({ }_{41}^{0} R\right)^{-1} \times{ }_{6}^{0} R \text {. }
$$

Equation (9) shows the relation between the rotation matrix of the frame $\{41\}$ and the general rotation matrix which leads to the last three joint angles in each leg. Equation (9) can be expressed as follows:

$$
\left({ }_{6}^{41} R\right)_{\text {right }}=\left[\begin{array}{lll}
r_{11} & r_{12} & r_{13} \\
r_{21} & r_{22} & r_{23} \\
r_{31} & r_{32} & r_{33}
\end{array}\right],
$$

where all components $r_{\mathrm{ij}}$ are provided in (A2) for the right leg. The only exceptions to the assumed notation for $\mathrm{c}$ and $\mathrm{s}$ are $c_{6+\pi / 4}$ and $s_{6+\pi / 4}$ which are replaced with $\cos \left(\theta_{6}+\right.$ $\pi / 4)$ and $\sin \left(\theta_{6}+\pi / 4\right)$ respectively. The corresponding rotation matrix for the left leg is mathematically represented by:

$$
\left({ }_{6}^{41} R\right)_{\text {left }}=\left[\begin{array}{lll}
l_{11} & l_{12} & l_{13} \\
l_{21} & l_{22} & l_{23} \\
l_{31} & l_{32} & l_{33}
\end{array}\right],
$$

where $l_{\mathrm{ij}}$ are provided in (A3). Solving (9) using (10) and (11) leads to the closed formulations for the angles $\theta_{4}, \theta_{5}$, and $\theta_{6}$ provided in the Appendix for both legs. The Nao biped robot has HipYawPith joints for both legs which are mechanically connected together and driven by an actuator. This fact enforces equations to have similar values for the sixth joint angle considering the assumed positive direction for both frames of the left and right legs. After simplification, one may write final values for both leg joint angles as:

$$
\left(\theta_{6}\right)_{R}=\left(\theta_{6}\right)_{L}
$$

$$
=\frac{1}{2}\left(\tan ^{-1}\left(\frac{r_{21}}{r_{22}}\right)_{R}+\tan ^{-1}\left(\frac{l_{21}}{l_{22}}\right)_{L}\right)+\frac{\pi}{4} .
$$

In practice, rotations of the Nao joints are limited. This is important for the controller design since large values, which are at the margins, may lead to an unstable gait.

\section{B. Foot and torso trajectories:}

As mentioned in the previous section, input to the walk engine and inverse kinematic model is foot and torso trajectories. These trajectories could be specified on-line or off-line according to the nature of the biped robot and its computational capabilities. On-line trajectory generation is performed in human gait because of our remarkable abilities in gait optimization (for minimum energy consumption) and accessibility of the environmental data gathered by human perception system. This method of trajectory generation is not proper for many conventional biped robots such as the Nao.

Alternatively, off-line techniques are also studied and employed extensively because of their suitability for robots with lower processing abilities. Some of these methods are the human trajectories [17], Inverted Pendulum [16], and ZMP. The energy-optimized human trajectories approach [17] with captured human motion and related joint movements may not be suitable for all biped robot applications because of the difference in mass and inertia distribution between humans and robots. On the other hand, ZMP method is used in such a way that foot trajectory is generated off-line and hip (or torso) trajectory will be produced on-line in order to satisfy the stability criteria using the robot's feedback. The latter approach is utilized in this paper and foot trajectories are generated offline. The trajectories considered for the dynamic modeling are derived with the assumptions of $8 \mathrm{~cm} / \mathrm{s}$ walking speed and a $4 \mathrm{~cm}$ step length. The robot's foot has movement in both $x$ and $z$ directions, but no movement in $y$ direction for a straight walk. Another essential issue to be considered for a path is that the foot shall contact the ground with zero velocity in order to avoid the impact. Implementing velocity and kinematic constraints in the $x$ and $z$ axes brings about (13) in which $L_{s}$ and $h_{s}$ stands for step length and step height, respectively. The input parameter $0<u<1$ is also the input parameter and symbolizes time:

$$
\begin{aligned}
& x_{f}=\left(u-\frac{\sin (2 \pi u)}{2 \pi}\right) L_{s}, \\
& z_{f}=\frac{1}{2}(1-\cos (2 \pi u)) h_{s} .
\end{aligned}
$$

The torso oscillates in the shape of a sinusoidal wave around the midpoint of the position of the robot's feet on the ground. This movement produces a stable walk with mass transfer on the supporting leg and facilitates keeping the ZMP within the foot contact area. Therefore, the torso trajectory in the $\mathrm{x}$ direction follows the same trajectory as the foot in (13); in y direction, it is considered to have a sinusoidal shape, and in the $\mathrm{z}$ direction, it is assumed to be a constant value of 0.195 , which is the hip joint height.

\section{Walk engine controller:}

A brief introduction of the examined controller on a linear inverted pendulum model in the walk engine is presented in this section. The produced difference between the foot and torso trajectories is the input for the inverse kinematics equations to calculate the joint angles. The controller is schematically illustrated in Fig. 3. 


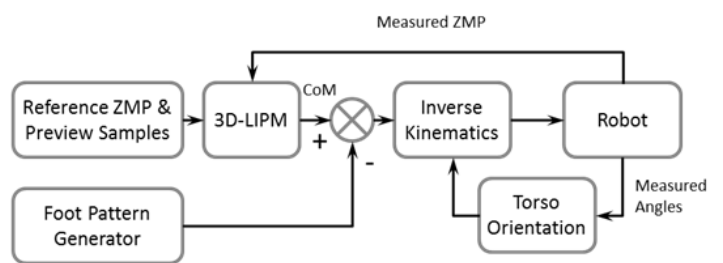

Fig. 3 Optimal preview control on the walk engine 3D-LIPM

Foot trajectories could be produced off-line according to pre-defined trajectories (13), but torso trajectories are generated consistent with the ZMP pulses for $\mathrm{N}$ future samples (in the preview controller). The developed perception [18], localization [19], and path planning [20] modules define robot and torso trajectories. Calculated torso trajectories, according to the future $\mathrm{N}$ samples of the ZMP, are employed with the measured ZMP in the 3DLIPM block to generate the CoM path for a stable walk.

\section{DYNAMIC MODELING}

Section II was introduced to outline the theoretical aspects of the kinematic modeling and trajectory generation. An accurate kinematic model, together with a preview controller on the 3D-LIPM and the ZMP trajectory leads to a stable walk. However, the proposed walk engine is not adaptable to various ground slopes and conditions. Developing torque controllers on necessitates an accurate and fast dynamic model to make a comparison between the predefined and feedback from the joints' torque and angle values. Furthermore, according to diverse walking maneuvers, an inverse dynamic study is required to size actuators coping with torque and power requirements. Therefore, the main objectives of the Nao robot's dynamic modeling in this section can be classified as: 1) estimation of the required torques for an actuator selection; and 2) facilitating the calculations for a threedimensional dynamic gait study and possible torque control approaches for future. This section includes developing three-dimensional dynamic modeling and modeling in the two separate sagittal and frontal planes. Discussed approach in this section results in an inverse dynamic problem with experimental data from joints as the input and required torques as the output. Five point masses are assumed for the dynamic model in the Cartesian coordinates as presented in Fig. 4. The assumed lower limb link pattern form an open loop serial chain, where the first rotation takes place in the supporting leg and the last one takes place in the non-supporting leg.

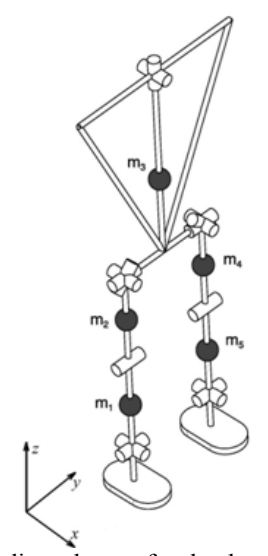

Fig. 4 Dedicated mass for the dynamic mode
The double support phase requires a hybrid dynamic model as studied in [21, 22]. However, this phase is not studied in this article and equations are derived with the assumption of a single support phase.

\section{A. Generalized dynamic modeling:}

Global positions of the links' center of masses are produced by the consecutive multiplication of the homogeneous transformation matrix (1) and local position vectors of $j^{\text {th }}$ mass, $L_{m j} \in \mathbb{R}^{4 \times 1}$, as mathematically described in by:

$$
\begin{gathered}
P_{m j}=\left(\prod_{i=1}^{n} T_{i}\right) L_{m j}, \\
P_{m j}=\left[\begin{array}{llll}
x_{m j} & y_{m j} & z_{m j} & 1
\end{array}\right]^{T},
\end{gathered}
$$

where $n$ is the number of joints before $j^{\text {th }}$ mass. The center of mass $(\mathrm{CoM})$ position vectors are provided in Table I for five dedicated mass points:

TABLE I. COM POSITION VECTORS

\begin{tabular}{c}
\hline Mass Center Position Vectors (mm); Five Masses \\
\hline$L_{\mathrm{m} 1}=[3.66,-1.52,38.72]^{\mathrm{T}}$ \\
$\mathrm{L}_{\mathrm{m} 2}=[-3.68,0.03,66.79]^{\mathrm{T}}$ \\
$\mathrm{L}_{\mathrm{m} 3}=[-4.80,50.06,127.27]^{\mathrm{T}}$ \\
$\mathrm{L}_{\mathrm{m} 4}=[-3.38,-0.03,-33.21]^{\mathrm{T}}$ \\
$\mathrm{L}_{\mathrm{m} 5}=[3.66,1.52,-64.03]^{\mathrm{T}}$ \\
\hline
\end{tabular}

Mass and inertia properties are also provided in Table A4 from the Nao's technical data sheet. Now, the chain rule is implemented in (14) to calculate the time derivatives of the position vectors:

$$
\begin{gathered}
\dot{P}_{m}=\frac{\partial P_{m}}{\partial t}=\frac{\partial P_{m}}{\partial q} \cdot \frac{\partial q}{\partial t}=J_{p} \dot{q}, \\
\ddot{P}_{m}=\frac{\partial J_{p}}{\partial q} \dot{q}^{2}+J_{p} \ddot{q},
\end{gathered}
$$

where $J_{\mathrm{p}}$ symbolizes the Jacobian matrix, which transforms the joint's angular velocities to the translational velocities of the masses. The position vector $\boldsymbol{q}$ is defined as:

$$
q=\left[\begin{array}{llll}
q_{1} & q_{2} & \cdots & q_{n}
\end{array}\right]^{T}, \quad n=12
$$

Acceleration and velocity vectors of point masses $\ddot{P}_{m}, \dot{P}_{m}$ are derived with respect to the supporting leg. The robot's equations of motion are developed in this section, using the Lagrange's approach on a kinematic chain with 12 degrees of freedom. Modified position vector $q \in \boldsymbol{Q}$ determines the robot's orientation and position in the global coordinates. Configuration space $\boldsymbol{Q}$ is introduced for the single support phase as well. Lagrangian is expressed as the difference between the system's kinetic energy $K$ and potential energy $V$ :

$$
\mathcal{L}(q, \dot{q})=K(q, \dot{q})-V(q)
$$

Equations of motion are then derived from (17) as follows:

$$
\frac{d}{d t} \frac{\partial \mathcal{L}}{\partial \dot{q}_{i}}-\frac{\partial \mathcal{L}}{\partial q_{i}}=f_{i}
$$

in which, $f_{i}=\sum_{j} \bar{F}_{j} \cdot \frac{\partial \bar{r}_{j}}{\partial q_{i}}$ with non-constraint forces $\bar{F}_{j}$ and positions $\bar{r}_{j}$ is introduced based on the generalized force definition associated with the generalized coordinates $q_{i}$. The torque exerted on links could be calculated after mapping the torques from the equation of motion to the 
actuators with the implementation of the Jacobian matrix. The skew-symmetric matrix, $\widehat{\omega}$, is introduced for angular velocity: $\quad \omega=\left[\begin{array}{lll}\omega_{1} & \omega_{2} & \omega_{3}\end{array}\right] \in \mathbb{R}^{3}$ and the unpacking operator is used to change the skew-symmetric matrix to the angular velocity vector as:

$$
\widehat{\omega}=\left[\begin{array}{ccc}
0 & -\omega_{3} & \omega_{2} \\
\omega_{3} & 0 & -\omega_{1} \\
-\omega_{2} & \omega_{1} & 0
\end{array}\right] ;(\widehat{\omega})^{\vee}=\omega .
$$

Multiplication of a rotation matrix inverse and its time derivative is a skew-symmetric matrix [23]. Thus, the time derivative of the rotation matrix could be expressed as:

$$
\dot{R}=R R^{-1} \dot{R}=R \widehat{\omega} \text {. }
$$

Angular velocity of each link in the previous joint frame is:

$$
\omega=\left(R^{-1} \dot{R}\right)^{\vee} \in \mathbb{R}^{3}
$$

Expansion of (20) can be expressed as:

$$
\widehat{\omega}(q, \dot{q})=\sum_{i=1}^{n} R^{-1}(q) \frac{\partial R(q)}{\partial q_{i}} \dot{q}_{i} ; \omega=J_{o} \dot{q},
$$

where $J_{o}$ symbolizes Jacobian matrix for angular velocities. Then, the kinetic energy of a link may be written as:

$$
K_{j}(q, \dot{q})=\frac{1}{2} \dot{q}^{T} \bar{D}_{j}(q) \dot{q},
$$

where $\bar{D}_{j}(q)$ is a symmetric, positive semi definite matrix:

$$
\bar{D}_{j}(q)=m J_{p}^{T}(q) J_{p}(q)+J_{o}^{T}(q) \boldsymbol{I}_{\text {rot }} J_{o}(q),
$$

in which $\boldsymbol{I}_{\text {rot }}$ represents the inertia tensor. The total kinetic energy of the system is:

$$
K(q, \dot{q})=\frac{1}{2} \dot{q}^{T} D(q) \dot{q},
$$

and $D(q)$ is defined as:

$$
D(q)=\sum_{j=1}^{N} \bar{D}_{j}(q)
$$

The potential energy is independent from $\dot{q}$ and is also attainable for $j^{\text {th }}$ mass with $z_{m j}$ as the robot's torso height; the total potential energy is the sum of the potential energy for all dedicated mass points with $g$ as the gravity acceleration:

$$
V(q)=\sum_{j=1}^{N} g m_{j} z_{m j}(q) .
$$

Expanding equation (18) results in:

$$
\frac{d}{d t} \frac{\partial K(q, \dot{q})}{\partial \dot{q}_{i}}-\frac{\partial K(q, \dot{q})}{\partial q_{i}}+\frac{\partial V(q)}{\partial q_{i}}=f_{i},
$$

that can be written as follows considering all lumped masses:

$$
\begin{aligned}
\sum_{j=1}^{n} D_{i j}(q) \ddot{q}_{j}+ & \sum_{j, k=1}^{n}\left(\frac{\partial D_{i j}(q)}{\partial q_{k}} \dot{q}_{j} \dot{q}_{k}\right. \\
& \left.-\frac{1}{2} \frac{\partial D_{i j}(q)}{\partial q_{i}} \dot{q}_{j} \dot{q}_{k}\right)+\frac{\partial V(q)}{\partial q_{i}}=f_{i}
\end{aligned}
$$

Therefore, the second term of (29) could be replaced by:

$$
\begin{gathered}
\sum_{j, k=1}^{n}\left(\frac{\partial D_{i j}(q)}{\partial q_{k}} \dot{q}_{j} \dot{q}_{k}-\frac{1}{2} \frac{\partial D_{i j}(q)}{\partial q_{i}} \dot{q}_{j} \dot{q}_{k}\right) \\
=\sum_{j=1}^{n} C_{i j} \dot{q}_{j},
\end{gathered}
$$

using Christoffel for vector notation and Coriolis matrix $C(q, \dot{q}) \in \mathbb{R}^{n \times n}$. The equivalent external forces on the system could be replaced with joint torques $\tau$ by neglecting the friction effect and in the single support state to reach the inverse dynamics equation:

$$
D(q) \ddot{q}+C(q, \dot{q}) \dot{q}+G(q)=\tau,
$$

where $G \in \mathbb{R}^{n}$ symbolizes potential energy and can be replaced by partial derivatives of (27) with respect to the position vector $q_{i}$. The double support condition will be considered separately as a future work of this study. Equation (31) contains inertia, Coriolis, centrifugal, and gravitational effects. Equation (31) can be rewritten as:

$$
\begin{gathered}
\dot{x}=\left[\begin{array}{c}
\dot{q} \\
D^{-1}(q)[-C(q, \dot{q}) \dot{q}-G(q)+\tau]
\end{array}\right], \\
\dot{x}=\mathcal{F}(x)+\mathcal{G}(x) u,
\end{gathered}
$$

with states $x=\left[\begin{array}{ll}q & \dot{q}\end{array}\right]^{T}$. Moreover, $\mathcal{G}(x)$ is independent from $\dot{q}$ in the natural coordinates $\left[\begin{array}{ll}q & \dot{q}\end{array}\right]^{T}$. The joint angular velocity and acceleration values are estimated with the numerical differentiation of the input joint angles, a sampling time $40 \mathrm{mSec}$, and a proper low-pass filter. This study is an inverse dynamics analysis since experimental joins data is available and performs as input to the model. Fig. 5 represents the right leg joint angles and serves as the input to the inverse dynamics equation where the right single support leg is denoted by SSL Right.
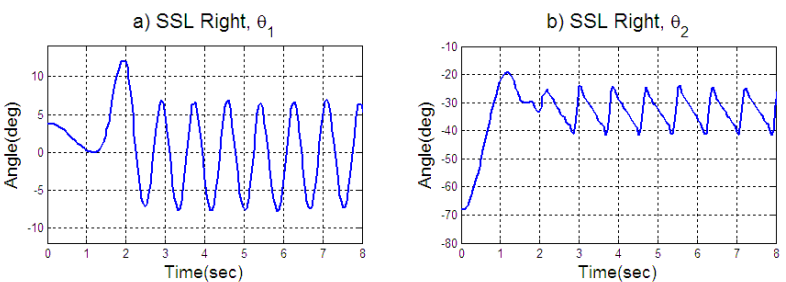

c) SSL Right, $\theta$
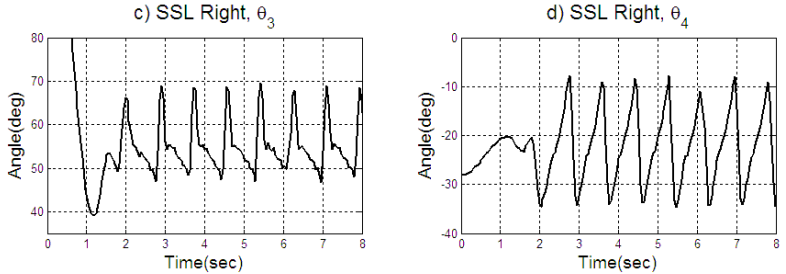

Fig. 5 Right leg's sensory data for straight walk of the Nao

Estimated torque values from the inverse dynamic equation (31) are provided in the "Results and Discussion" section. The computational cost of the general 3D dynamic modeling prompts us to develop a simplified model with lower calculations such as dynamic study in two separate sagittal and frontal planes [24].

\section{B. Modeling in the sagittal and frontal planes:}

This sub-section contributes to decompositions in independent sagittal and frontal planes. Joints 2, 3, and 4 on the right foot and 9,10, and 11 on the left foot are considered in the sagittal study, thus there are six degrees of freedom for modeling in this plane. The homogenous 
transformation (1) changes to the following form for the sagittal plane $T_{s, i} \in \mathbb{R}^{4 \times 4}$ :

$$
T_{s, i}=\left[\begin{array}{cc}
R_{s, i} & L_{s, i} \\
O_{1 \times 3} & 1
\end{array}\right]
$$

in which $L_{s, i} \in \mathbb{R}^{3 \times 1}$ describes the position vector of the $i^{\text {th }}$ joint with respect to the $i^{\text {th }}-1$ joint. Similarly, the local position vector of mass $j^{t h}, L_{m s, j} \in \mathbb{R}^{4 \times 1}$ will be obtained by setting the second component of corresponding vector $L_{m j}$ to zero. As a result, the global position vector of point masses is accessible in the $(x-z)$ plane as:

$$
\begin{gathered}
P_{m s, j}=\left(\prod_{i=1}^{n} T_{s, i}\right) L_{m s, j}, \\
P_{m s, j}=\left[\begin{array}{llll}
x_{m s, j} & 0 & z_{m s, j} & 1
\end{array}\right]^{T},
\end{gathered}
$$

where $n$ shows the joints before the dedicated point mass $j^{t h}$. The modified position vector $q_{s} \in \boldsymbol{Q}_{\boldsymbol{s}}$ shows the position and the orientation of the robot in the sagittal plane.

$$
q_{s}=\left[\begin{array}{llll}
q_{s 1} & q_{s 2} & \cdots & q_{s n}
\end{array}\right]^{T}, \quad n s=6
$$

The equation of motion (31) changes to the following form in the sagittal plane:

$$
D_{s}\left(q_{s}\right) \ddot{q}_{s}+C_{s}\left(q_{s}, \dot{q}_{s}\right) \dot{q}_{s}+G_{s}\left(q_{s}\right)=\tau_{s},
$$

where $D_{s}\left(q_{s}\right) \in \mathbb{R}^{\mathrm{ns} \times \mathrm{ns}}, C_{s}\left(q_{s}, \dot{q}_{s}\right) \in \mathbb{R}^{\mathrm{n} s \times \mathrm{ns}}, G_{s} \in \mathbb{R}^{\mathrm{ns}}$, and $\tau_{s} \in \mathbb{R}^{\mathrm{ns}-1}$. Therefore, the state-space notation of the equation of motion in the sagittal plane for the single support phase yields:

$$
\begin{gathered}
\dot{x}_{s}=\left[\begin{array}{c}
\dot{q}_{s} \\
D_{s}{ }^{-1}\left(q_{s}\right)\left[-C_{s}\left(q_{s}, \dot{q}_{s}\right) \dot{q}_{s}-G_{s}\left(q_{s}\right)+\tau_{s}\right]
\end{array}\right] \\
\dot{x}_{s}=\mathcal{F}_{s}\left(x_{s}\right)+\mathcal{G}_{s}\left(x_{s}\right) u_{s},
\end{gathered}
$$

where $\quad x_{s}=\left[\begin{array}{ll}q_{s} & \dot{q}_{s}\end{array}\right]^{T}$ and $\mathcal{G}_{s}\left(x_{s}\right) \in \mathbb{R}^{2 n s \times(n s-1)}$ Alternatively, joints 1 and 5 on the right foot and joints 8 and 12 on the left foot are considered in the (y-z) plane. The AnkleRoll joint of the non-supporting leg could be eliminated from the equations, since it has no effect on the robot's motion when it has no ground contact. Similar to the sagittal plane approach, the homogenous transformation for the $(\mathrm{y}-\mathrm{z})$ plane is:

$$
T_{f, i}=\left[\begin{array}{cc}
R_{f, i} & L_{f, i} \\
O_{1 \times 3} & 1
\end{array}\right] .
$$

The local position vector of the mass $j^{t h}, L_{m f, j} \in$ $\mathbb{R}^{4 \times 1}$ is attainable by setting the first component of the corresponding vector $L_{m j}$ to zero. Then, the global position vector of the point masses in the frontal plane is expressed by the successive multiplication of the homogeneous transformations and the local position vector of the corresponding mass:

$$
\begin{gathered}
P_{m f, j}=\left(\prod_{i=1}^{n} T_{f, i}\right) L_{m f, j}, \\
P_{m f, j}=\left[\begin{array}{llll}
0 & y_{m f, j} & z_{m f, j} & 1
\end{array}\right]^{T},
\end{gathered}
$$

where $z_{m f, j}=z_{m s, j}$. The frontal plane's equation of motion could be written as:

$$
D_{f}\left(q_{f}\right) \ddot{q}_{f}+C_{f}\left(q_{f}, \dot{q}_{f}\right) \dot{q}_{f}+G_{f}\left(q_{f}\right)=\tau_{f},
$$
where $D_{f}\left(q_{f}\right) \in \mathbb{R}^{\mathrm{nf} \times \mathrm{nf}}, C_{f}\left(q_{f}, \dot{q}_{f}\right) \in \mathbb{R}^{\mathrm{n} f \times \mathrm{nf}}, G_{f} \in \mathbb{R}^{\mathrm{nf}}$, and $\tau_{f} \in \mathbb{R}^{\mathrm{nf}-1}$. The position vector $q_{f} \in \mathcal{Q}_{f}$ shows the general formation of the open kinematic chain in the frontal plane.

$$
q_{f}=\left[\begin{array}{llll}
q_{1 f} & q_{2 f} & \cdots & q_{n f}
\end{array}\right]^{T}, \quad n f=6
$$

As a result, the corresponding state-space notation with $x_{f}=\left[\begin{array}{ll}q_{f} & \dot{q}_{f}\end{array}\right]^{T}$ is described as:

$$
\begin{aligned}
& \dot{x}_{f}=\left[\begin{array}{c}
\dot{q}_{f} \\
D_{f}{ }^{-1}\left(q_{f}\right)\left[-C_{f}\left(q_{f}, \dot{q}_{f}\right) \dot{q}_{f}-G_{f}\left(q_{f}\right)+\tau_{f}\right]
\end{array}\right] \text {, } \\
& \dot{x}_{f}=\mathcal{F}_{f}\left(x_{f}\right)+\mathcal{G}_{f}\left(x_{f}\right) u_{f},
\end{aligned}
$$

where $\mathcal{G}_{f}\left(x_{f}\right) \in \mathbb{R}^{2 n f \times(n f-1)}$. The estimated torques from the inverse dynamics approach in $(\mathrm{x}-\mathrm{z})$ and $(\mathrm{y}-\mathrm{z})$ planes are both provided in Section IV.

\section{Modeling in a multi-body dynamics package:}

The 3D lower body of the selected Nao platform is modeled in MSC Adams. This was done in order to verify the analytic inverse dynamic approach in the previous subsection. All the physical characteristics are plugged into the new model in MSC Adams with five lumped masses. Firstly, the single support phase with a step length of $4 \mathrm{~cm}$ and a speed of $8 \mathrm{~cm} / \mathrm{s}$ is modeled. The developed model for the fixed condition of the supporting leg is schematically depicted in Fig. 6.
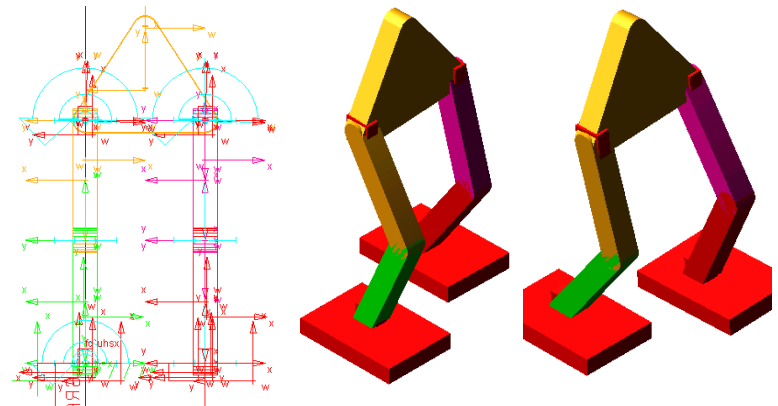

Fig. 6 MSC Adams model of the Nao biped robot in walking

All joints are modeled according to the Nao data sheet to accomplish Roll and Pitch duties as it is shown in Fig. 7. This figure shows four slides within a straight step action.

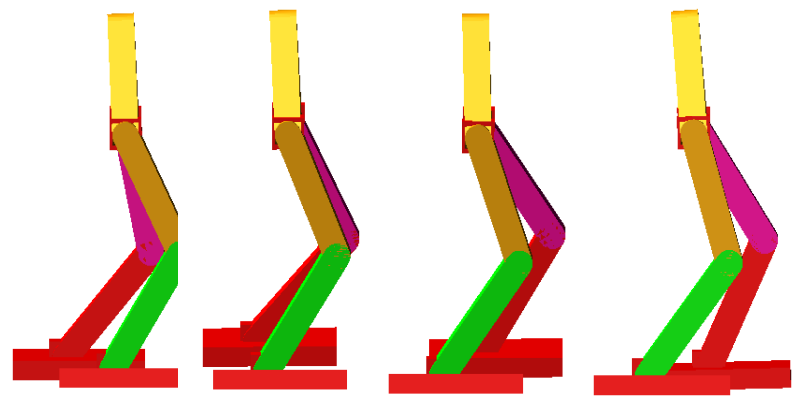

Fig. 7 Walking stages of the Nao with right leg as supporting leg

The degree of freedom for this model is ten since the HipYawPitch joint is ignored similar to the general 3D dynamic modeling in the previous sub-section. All these ten degrees of freedom were changed to inputs and replaced with real joint angles for a straight walk. The ground condition is also considered as the second simulation part with a contact model. This will be described in the next section. 


\section{RESUltS AND DiscUSSION}

Several simulations were carried out in this section on the proposed kinematic and dynamic modeling of the Nao robot to verify the approach. The first set of analysis in this section examined the performance of the inverse kinematic approach, which results in tangible joint angle data (as graphically illustrated in Fig. 8). This walk is performed with a step length of $4 \mathrm{~cm}$, a speed of $8 \mathrm{~cm} / \mathrm{s}$, and foot trajectories (13). The curves show that the KneePitch joint angle for the left leg has a minute value and could be neglected from dynamic study to reduce the on-line load of computation.
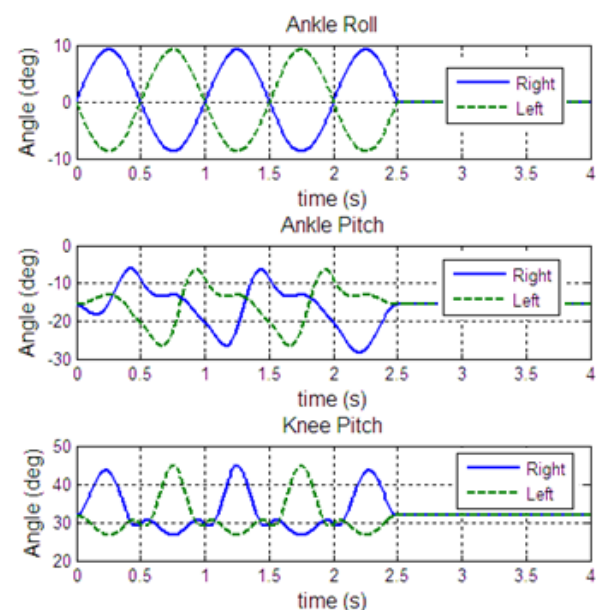

Fig. 8 Joint angle data for the first three joints of both legs produced by the inverse kinematics

The estimated joint angles from the developed inverse kinematics code for the remaining three joints of the right leg are provided in Fig. 9.

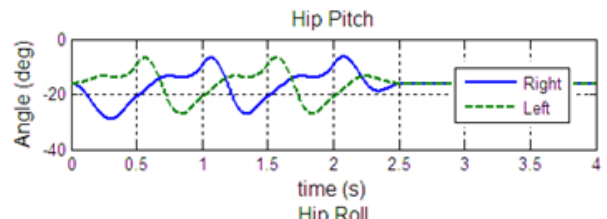
Hip Roll

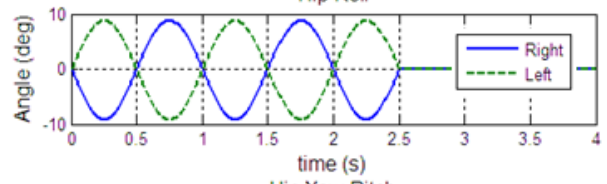

Hip Yaw-Pitch

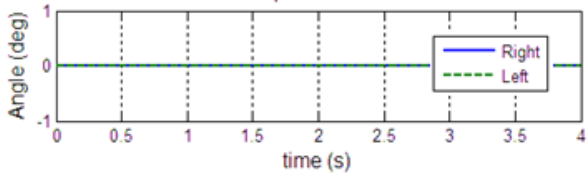

Fig. 9 HipPitch, HipRoll, and HipPitch joint angle data by inverse kinematics for both legs in the open-loop walk engine

These values are within the allowable margins of the robot's joint angles. Estimated torque results in Fig. 10 are obtained with the inverse dynamics (32) and real sensory data provided in Fig. 5.
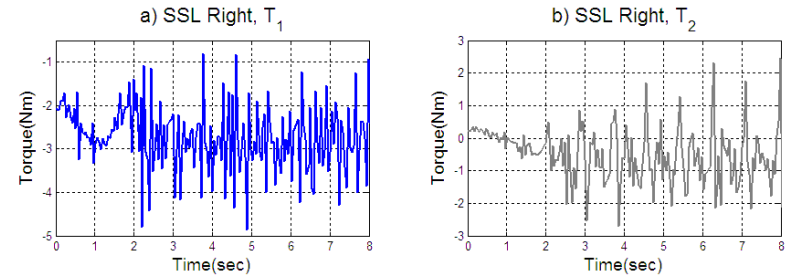

c) SSL Right, $T_{3}$

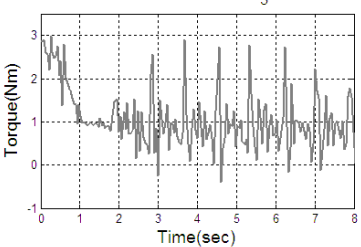

d) SSL Right, $T_{4}$

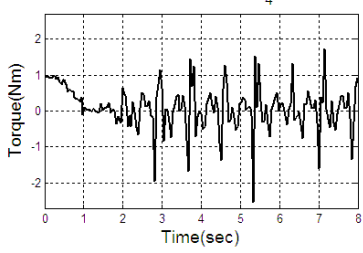

Fig. 10 Torques of the AnkleRoll, AnklePitch, KneePitch, and HipPitch joints on the right leg

The same approach is applied for torque estimation of joints 5, 6, 7, and 8 in Fig. 11. Estimated torques for each joint using the measured joint angles stands within the allowable torque margins of the Nao biped robot.
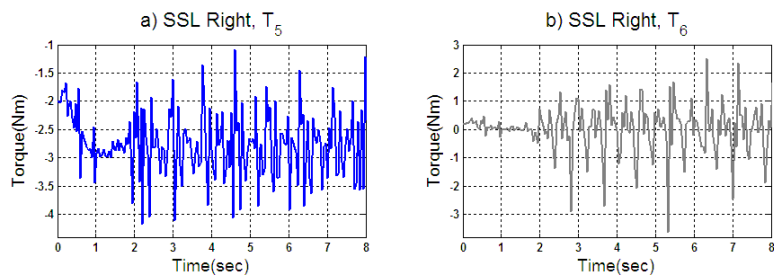

c) SSL Right, $T_{7}$
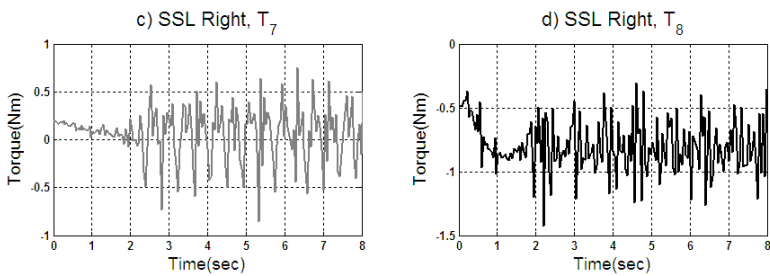

Fig. 11 Required torques of the right HipRoll, right HipYawPitch, left HipYawPitch, and left HipRoll joints

Fig. 12 provide a comparison between the MSC Adams inverse dynamic simulation and the proposed modeling approach.
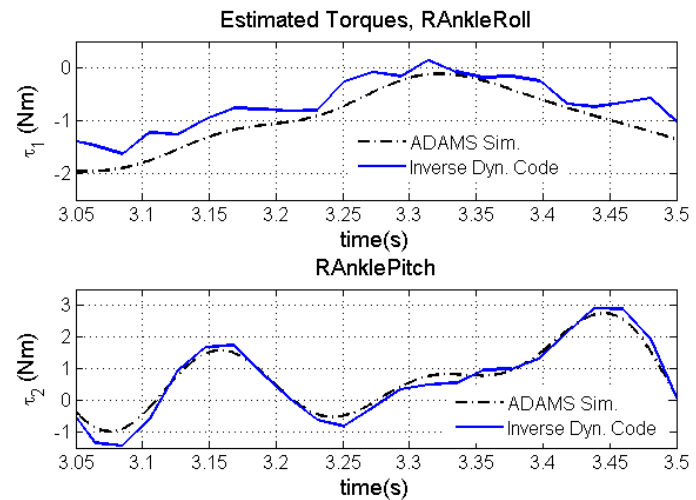

Fig. 12 Simulation data vs. ADAMS output of the supporting leg

Selected Right AnkleRoll and Right AnklePitch joints bear the largest torque values during lift off of the left leg. These curves presented only for a $0.5 \mathrm{sec}$ period (which is the duration of one step for the left leg).

The observed errors are for the right AnkleRoll joint $\tau_{1}$, especially at the beginning of the left leg's motion. For 
the purpose of analysis, the other joints results (which have lower torque values) are compared in Fig. 13 and Fig. 14.

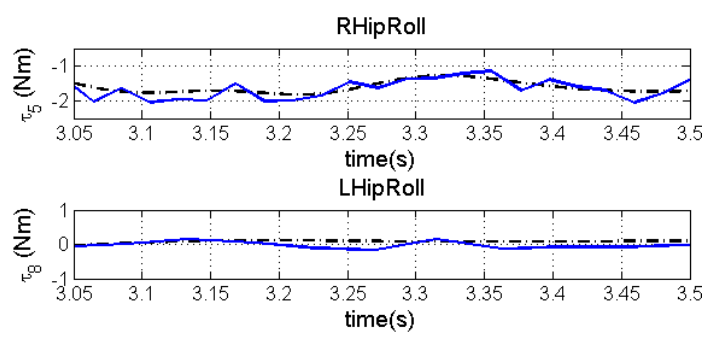

Fig. 13 Comparison between MATLAB code and ADAMS simulation data for the fixed supporting leg condition

Estimated torques from the proposed methodology and MSC Adams are compared in Fig. 14 for the AnklePitch of the non-supporting leg.

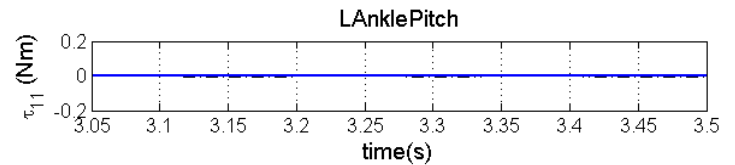

Fig. 14 Verification of results with ADAMS for left leg joints

This is promising for using the inverse dynamic model for the fixed-to-ground condition of the supporting leg, which makes the control more straightforward and flexible to various trajectory requirements. In addition, the robot's center of mass position from the MSC Adams model is provided for a half step length $(4 \mathrm{~cm})$ in Fig. 15.
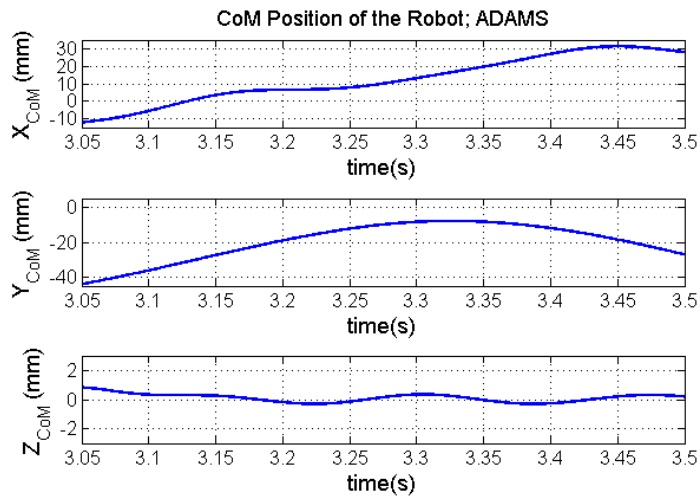

Fig. 15 Verification of CoM position generated by ADAMS

Another implication of the findings from Fig. 13 and Fig. 15 is that the proposed inverse kinematic formulations perform well in the presence of various generated trajectories.

The second part of this section is dedicated to the consideration of ground contact to investigate the effect of ground characteristics. To perform ground contact modeling of the robot's foot contact, the "Impact" and "Restitution" models of the MSC Adams package are examined. This analysis includes several contact condition including changing Stiffness, Damping, Penetration Depth, and Force Exponent in the "Impact" model, as well as changing Penalty parameter and Restitution Coefficient in the "Restitution" model. After several testing and tuning parameters, the impact model with the consideration of Coulomb friction model (static coefficient 0.41, dynamic coefficient 0.27 ) is employed and torque results are plotted in Fig. 16. These tests include verification of the model with the real Nao robot platform to identify parameters. Various tests are performed in the simulation package with different impact parameters to have a stable walk with the same speed and with the same joint angles (as the input to the inverse dynamic model). Afterward, the parameters that lead to the same output are identified for the simulation with the recursive least square method. Fig. 16 represents the first joint's (right AnkleRoll) torque in three directions from the MSC Adams simulations.

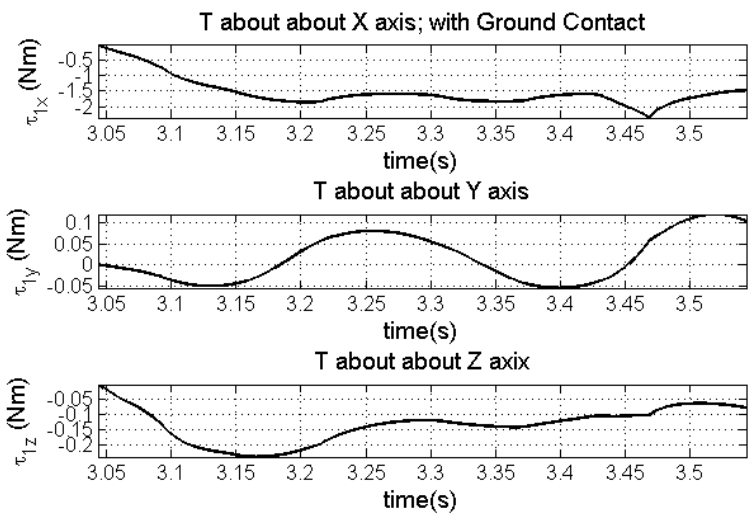

Fig. 16 Imposed torques on the first joint with ground contact

These curves substantiate the effects of ground contact and lateral friction, which lead to the presence of torques in two other axes of the joint. Larger values of torques are contributed to the AnkleRoll, especially before the final stages of the non-support leg contacting the ground. Other supporting leg's joint torques with ground contact considerations are shown in Fig. 17 and Fig. 18.

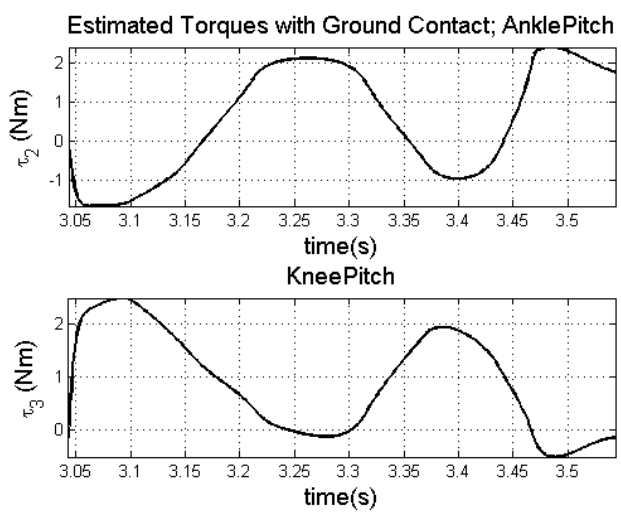

Fig. 17 AnklePitch \& KneePitch estimated torques; ADAMS model with contact
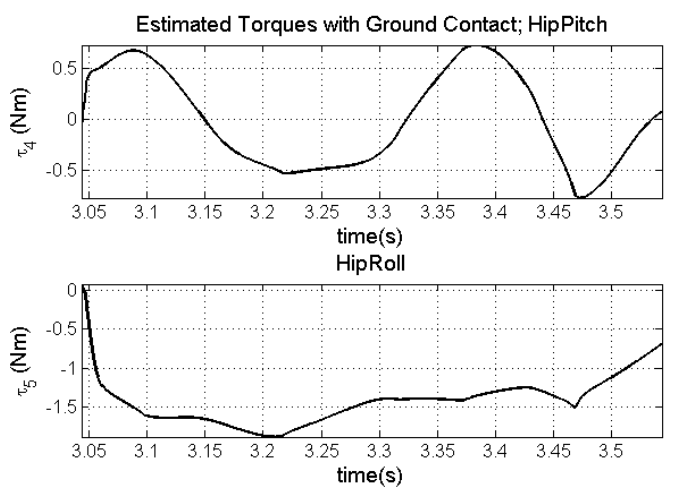

Fig. 18 HipPitch \& HipRoll estimated torques; ADAMS model with contact 
The type of solver plays an important role in the "Dynamic" solution of such problems in MSC Adams. This issue is also tested with various dynamic solvers and accurate results were extracted from the "SI2" (StabilizedIndex-2) solver instead of conventional dynamic solvers. This solver reduces the original index 3 DEA (Differential Algebraic Equation) multibody dynamic problem in mechanical systems to an analytically equivalent index problem. This is done with solving the velocity kinematic constraints together with the kinematic differential equations. The SI2 method is characteristically slower than the index 3 method, but provides more accurate outcomes. It is also observed that the conventional dynamic solvers are sensitive to ground condition changes, which results in large calculated torque values. The integrator effect is also studied; it is noted that the "GSTIFF" produce more stable and smooth results, with lower oscillations in the presence of the changing time step, in comparison with the "WSTIFF". Various ground contact condition has been investigated in the multibody dynamics package and will be incorporated in the proposed analytical inverse dynamic methodology.

\section{CONCLUSIONS}

The presented approach and modeling is tested on the Nao biped robot and shows a reasonable performance during a straight walk. This paper focuses on the dynamic modeling of the Nao with assumptions of the predefined foot trajectories and the generated on-line torso trajectories by the walk engine stability controller. The procedure of comprehensive kinematic modeling of the robot, which facilitates joint angles determination, velocity, and acceleration for different dedicated trajectories, are introduced. The inverse dynamics results were verified with the estimated torques of the MSC Adams packages for the same trajectory planning and input joint angles. Therefore, the proposed dynamic modeling approach can be used for dependable torque control techniques to cope with various ground conditions.

Improper ground contact selection has been identified as major contributing factor for torque estimation errors up to $54 \%$. More studies need to be done to establish a "User Defined" ground contact to model nonlinear behavior and characteristics of the biped robot's foot contact. Supplementary studies need to be performed to establish a volume-based ground contact model for the selected biped robot performing a stable walk.

\section{REFERENCES}

[1] Grizzle, J. W., Chevallereau, C., Ames, A. D., and Sinnet, R. W., "3D Bipedal robotic walking: models, feedback control, and open problems," In 8th IFAC Symposium on Nonlinear Control Systems, Bologna, Italy, 2010.

[2] Wollherr, D., Buss, M., Hardt, M., Stryk, O. V., "Research and development towards an autonomous biped walking robot," In Proc. of the IEEE /ASME Int. Conf. on Advanced Intelligent Mechatronics, AIM, 2003.

[3] Fujimoto, Y., "Trajectory generation of biped running robot with minimum energy consumption," In Proc. of the IEEE Int. Conf. on Robotics \& Automation, pp. 3803-3808, 2004.

[4] Huang, O., Yokoi, K., Kajita, S., Kaneko, K., Arai, H., Koyachi, N., Tanie, K., "Planning Walking Patterns for a Biped Robot," IEEE Transactions on Robotics and Automation, Vol. 17, pp. 280 289, 2001.

[5] Ghaffari Jadidi, M., Hashemi, E., Zakeri Harandi, M.A., Sadjadian, H., "Kinematic modeling improvement and trajectory planning of the nao biped robot," In Proc. of Joint Int. Conf. on Multibody System Dynamics, Finland, 2010.
[6] Azevedo, C., Andreff, N., Arias, S., "Bipedal Walking: From Gait Design to Experimental Analysis. Mechatronics," Elsevier, Vol. 14, No. 6, 639-665, 2004.

[7] Hurmuzlua, Y., Genot, F., Brogliatoc, B., "Modeling, Stability and Control of Biped Robots-A General Framework," Automatica, Vol. 40, pp. 1647 - 1664, 2004.

[8] Kajita, S., Kanehiro, F., Kaneko, K., Fujiwara, K., Harada, K., Yokoi, K., Hirukawa, H., "Biped Walking Pattern Generation by using Preview Control of Zero-Moment Point," In Proc. of the IEEE Int. Conf. on Robotics\& Automation, 2003.

[9] Kajita, S. Kanehiro, K., Kaneko, K., Fujiwara, K., Yokoi, K. and Hirukawa, H., "A realtime pattern generator for biped walking," Proc. of the 2002 IEEE International Conference on Robotics and Automation, Washington D.C., pp. 31-37, 2002.

[10] Kajita, S., Morisawa, M., Harada, K., Kaneko, K., Kanehiro, F., Fujiwara, K., Hirukawa, H., "Biped Walking Pattern Generator Allowing Auxiliary ZMP Control," In Proc. of the IEEE/RSJ Int. Conf. on Intelligent Robots and Systems, pp. 2993-99, 2006.

[11] Craig, John J. Introduction to Robotics: Mechanics and Control, Pearson Prentice Hall, 3rd edition, 2005.

[12] Jens Christensen, Jesper Lundgaard Nielsen, Mads Sølver Svendsen, and Peter Falkesgaard Ørts. Development, Modeling And Control of A Humanoid Robot, Master's thesis, Aalborg University, 2007.

[13] Spong, M. W., Hutchinson, S. and Vidyasagar, M. Robot Modeling and Control, 1st Edition, John Wiley \& Sons, Inc, 2005.

[14] Goldenberg, A. A, Benhabib, B., and Fenton, R. G. "A Complete Generalized Solution to the Inverse Kinematics of Robots," IEEE Journal of Robotics and Automation, RA-1(1), 14-20, (1985).

[15] Hashemi, E. and Jadidi, M.G., "Dynamic Modeling and Control Study of the NAO Biped Robot with Improved Trajectory Planning," Ch. 42, 1-18, Book ID 270_694_1_En, Springer, ISBN 978-3-642-22699-1, 2011

[16] Kajita, S. , Matsumotq, O., and Saigo. M. "Real-time 3D walking pattern generation for a biped robot with telescopic legs," In Proc. of the 2001 IEEE International Conference on Robotics and Automation, pp. 2299-2308, 2001.

[17] Pedersen, M. M., Nielsen, A. A. and Christensen, L. F. Design of Biped Robot AAU-BOT1. Master's thesis, Aalborg University, 2007.

[18] Hashemi, E., Jadidi, M.G., Yaghobi, M., Shafiei, M.R.; Lashgarian, M., ShahMohammadi, M.R., Faraji, F., Zareie, K., "MRL-SPL Team Description 2012 Standard Platform League," RoboCup, 2012.

[19] Hashemi, E., Jadid, M. G., Lashgarian, M., Yaghobi, M., \& Shafiei, R. M. "Particle filter based localization of the nao biped robots," inn System Theory (SSST), 2012 44th Southeastern Symposium on, pp. 168-173, 2012.

[20] Hashemi, E., Jadidi, M.G., ShahMohamamdi, M.R., Karimi, M., "In-Plane Path Planning for Biped Robots Based on Bezier Curve," Advanced Intelligent Mechatronics (AIM), IEEE/ASME International Conference on, pp. 796-801, 2011.

[21] Westervelt, E.R.; Grizzle, J.W.; Koditscheky, D.E. "Hybrid Zero Dynamics of Planar Biped Walkers," IEEE Trans. on Automatic Control, Vol. 48, pp. 42-56, 2003.

[22] Mu, Xiuping, and Qiong Wu. "Development of a complete dynamic model of a planar five-link biped and sliding mode control of its locomotion during the double support phase." International J. of Control Vol. 77, No. 8, pp. 789-799, 2004.

[23] Murray, R. M., Li, Z. and Sastry, S. A Mathematical Introduction to Robotic Manipulation. CRC Press, 1994.

[24] Jadidi, M.G., Sadjadian, H., Hashemi, E. Shahmohammadi, M. R., "Three-Dimensional Dynamic Modeling of the Nao Biped Robot with Simplified Equations of Motion in Sagittal and Frontal Planes," in Proc. of Joint International Conf. on Multibody System Dynamics, 2012

\section{NOMENCLATURE}

\begin{tabular}{cl}
\hline Symbol & \multicolumn{1}{c}{ Description } \\
\hline$A_{i}$ & Homogenous transf. between the joints \\
& $i^{t h}$ and $i^{t h}-1$ \\
$a_{i}, d_{i}$ & DH parameters (distance) \\
$C(q, \dot{q})$ & Coriolis matrix
\end{tabular}




\begin{tabular}{|c|c|}
\hline$C, s$ & $\cos$ and sin functions \\
\hline$D$ & Generalized inertia matrix \\
\hline $\bar{D}$ & Positive semi def. bodies' inertia matrix \\
\hline$f, g$ & Functions of DH parameters \\
\hline$f_{i}$ & Non-conservative forces/joint torques \\
\hline $\bar{F}_{i}$ & associated with the gen. Coord. $q_{i}$ \\
\hline $\begin{array}{c}I_{j} \\
G(q)\end{array}$ & Potential eneroy vector \\
\hline$h(4)$ & Potential energy vector \\
\hline$I_{\text {rot }}$ & $\begin{array}{l}\text { Foot trajectory step height } \\
\text { Inertia tensor }\end{array}$ \\
\hline$K$ & Kinetic energy \\
\hline $\begin{array}{c}l_{m n} \\
L_{i}\end{array}$ & $\begin{array}{l}\text { Left leg rot. matrices components } \\
\text { position vector of the } i^{t h} \text { w.r.t } i^{t h}-1\end{array}$ \\
\hline$L_{m k}$ & Mass centers' position vectors \\
\hline$L_{s}$ & Foot trajectory step length \\
\hline$m_{j}$ & Mass point $j$ \\
\hline$P_{j}^{i}$ & Position vector $j$ rel. to $i$ \\
\hline$P_{m}$ & Robot's mass centers position matrix \\
\hline$q$ & Robot's pos. matrix in the global Coord. \\
\hline $\bar{r}_{j}$ & Positions of non-conservative forces \\
\hline$R_{i}$ & $\begin{array}{l}\text { Base rotation matrix of the } i^{t h} \text { joint about } \\
\text { its rotation axes }\end{array}$ \\
\hline$R_{a, b}$ & $\begin{array}{l}\text { Rotation matrix about the } a \text { axis with } \\
\text { angle } b\end{array}$ \\
\hline${ }_{j}^{i} R$ & Rotation matrix $j$ relative to $i$ \\
\hline$r_{m n}$ & $\begin{array}{l}\text { Right leg rot. matrices components } \\
\text { Homogeneous transformation } j \text { rel. to } i\end{array}$ \\
\hline $\bar{T}$ & Translations matric in Cartesian Coord. \\
\hline$u$ & Input parameter for the foot trajectory \\
\hline$V$ & Potential and kinetic energy \\
\hline$x_{f}, y_{f}, z_{f}$ & Foot trajectory components \\
\hline$z_{m j}$ & Vertical position of the mass point $m_{j}$ \\
\hline$\alpha_{i}, \theta_{i}$ & DH parameters (angle) \\
\hline$\omega$ & Angular velocity vector \\
\hline$\widehat{\omega}$ & Skew-symmetric angular Vel. matrix \\
\hline$\tau$ & Joint's torque \\
\hline$(\cdot)_{L},(\cdot)_{R}$ & Left/right rotation/position matrices \\
\hline$(\cdot)_{f},(\cdot)_{s}$ & Rotation \& pos. matrices in frontal/sagittal \\
\hline$m f, j,(\cdot)_{m s, j}$ & Pos. vectors of mass $j$ in frontal/sagittal \\
\hline$(\cdot)_{f},(\cdot)_{s}$ & Rotation \& pos. matrices in frontal/sagittal \\
\hline
\end{tabular}

\section{APPENDIX A}

Mechanical and geometrical data mentioned in this article have already been extracted from the Nao technical specifications provided by the Aldebaran Co.

Axes of rotations of the studied Nao robots are provided in Tables A1.

TABLE A1. Dedicated AXes of Rotation For EACH Leg

\begin{tabular}{ccc}
\hline Joint & Rot. Axis & Name \\
\hline 1,5 & $\mathrm{x}$ & HipRoll, AnkleRoll \\
$2,3,4$ & $\mathrm{y}$ & HipPitch, KneePitch, Ankle Pitch \\
6 & $\mathrm{y}-\mathrm{z}$ & HipYawPitch \\
\hline
\end{tabular}

The DH parameters for the inverse kinematics and the dynamic are tabulated in Table A2 as follows:

TABLE A2. LEFT LEG DH PARAMETERS

\begin{tabular}{llllll}
\hline Frame & $\alpha_{i}$ & $a_{i}$ & $d_{i}$ & $\theta_{i}$ \\
\hline
\end{tabular}

\begin{tabular}{ccclc}
\hline (Joint) & \multicolumn{1}{c}{0} \\
\hline $\mathbf{1}$ & $-\pi / 2$ & 0 & 0 & $\theta_{1}$ \\
$\mathbf{2}$ & $\pi / 2$ & 0 & 0 & $\theta_{2}$ \\
$\mathbf{3}$ & 0 & Tibia length & 0 & $\theta_{3}$ \\
$\mathbf{4 0}$ & 0 & Thigh length & 0 & 0 \\
$\mathbf{4}$ & 0 & 0 & 0 & $\theta_{4}$ \\
$\mathbf{5}$ & $-\pi / 2$ & 0 & 0 & $\theta_{5}$ \\
$\mathbf{6}$ & $-\pi / 4$ & 0 & 0 & $\theta_{6}-3 \pi / 4$ \\
$\mathbf{4 1}$ & \multicolumn{5}{c}{$\operatorname{Rot}_{z}(\pi / 2) \times \operatorname{Rot}_{x}(\pi / 2)$} \\
$\mathbf{4 2}$ & $\operatorname{Rot}_{x}(-\pi / 2) \times \operatorname{Rot}_{z}(-\pi / 2)$ \\
$\mathbf{6 0}$ & $\operatorname{Rot}_{x}(\pi / 2) \times \operatorname{Rot}_{z}(\pi / 2)$ \\
\hline
\end{tabular}

Joint position vectors and the robot's inertial characteristics in the dynamic model are accessible from the Nao robots datasheet and are presented in Tables A3 and $\mathrm{A} 4$ below:

TABLE A3. JOINTS POSITION VECTORS

\begin{tabular}{l}
\hline Joint vector (mm) \\
\hline $\mathrm{L}_{1}=[0,0,0]^{\mathrm{T}}$ \\
$\mathrm{L}_{2}=[0,0,0]^{\mathrm{T}}$ \\
$\mathrm{L}_{3}=[0,0,102.75]^{\mathrm{T}}$ \\
$\mathrm{L}_{4}=[0,0,100]^{\mathrm{T}}$ \\
$\mathrm{L}_{5}=[0,0,0]^{\mathrm{T}}$ \\
$\mathrm{L}_{6}=[0,0,0]^{\mathrm{T}}$ \\
$\mathrm{L}_{7}=[0,100,0]^{\mathrm{T}}$ \\
$\mathrm{L}_{8}=[0,0,0]^{\mathrm{T}}$ \\
$\mathrm{L}_{9}=[0,0,0]^{\mathrm{T}}$ \\
$\mathrm{L}_{10}=[0,0,-100]^{\mathrm{T}}$ \\
$\mathrm{L}_{11}=[0,0,-102.75]^{\mathrm{T}}$ \\
$\mathrm{L}_{12}=[0,0,0]^{\mathrm{T}}$ \\
\hline
\end{tabular}

TABLE A4. MASS AND INERTIA PROPERTIES OF LINKS AND POINT MASSES

\begin{tabular}{rc}
\hline Mass (grams) & Inertia $\left(\mathbf{k g} \times \mathbf{~ m}^{\mathbf{2}}\right)$ \\
\hline $\mathrm{m}_{1}=435.98$ & {$\left[\mathrm{I}_{\mathrm{xx} 1}, \mathrm{I}_{\mathrm{yy} 1}, \mathrm{I}_{\mathrm{zz} 1}\right]=[0.0012,0.0012,0.0006]$} \\
$\mathrm{m}_{2}=605.72$ & {$\left[\mathrm{I}_{\mathrm{xx} 2}, \mathrm{I}_{\mathrm{yy} 2}, \mathrm{I}_{\mathrm{zz} 2}\right]=[0.0018,0.0018,0.0010]$} \\
$\mathrm{m}_{3}=1026.28$ & {$\left[\mathrm{I}_{\mathrm{xx} 3}, \mathrm{I}_{\mathrm{yy} 3}, \mathrm{I}_{\mathrm{zz} 3}\right]=[0.0049,0.0047,0.0016]$} \\
$\mathrm{m}_{4}=605.72$ & {$\left[\mathrm{I}_{\mathrm{xx} 4}, \mathrm{I}_{\mathrm{yy} 4}, \mathrm{I}_{\mathrm{zz} 4}\right]=[0.0018,0.0018,0.0010]$} \\
$\mathrm{m}_{6}=435.98$ & {$\left[\mathrm{I}_{\mathrm{xx} 5}, \mathrm{I}_{\mathrm{yy} 5}, \mathrm{I}_{\mathrm{zz} 5}\right]=[0.0012,0.0012,0.0006]$} \\
\hline
\end{tabular}

The AnkleRoll, AnkelPitch, and KneePitch angles are calculated using the trajectories as:

$$
\begin{aligned}
& \theta_{1}=\tan ^{-1}\left(\frac{y}{z}\right) \\
& \theta_{2}=-\tan ^{-1}\left(\frac{f_{2}\left(\theta_{3}\right)}{f_{1}\left(\theta_{3}\right)}\right)-\tan ^{-1}\left(\frac{x}{\sqrt{y^{2}+z^{2}}}\right) \\
& \theta_{3}=\cos ^{-1}\left(\frac{x^{2}+y^{2}+z^{2}-a_{4}^{2}-a_{3}^{2}}{2 a_{3} a_{4}}\right)
\end{aligned}
$$

Components of rotation matrices presented in (10) for the right leg are:

$$
\begin{aligned}
& r_{11}=-c_{6+\pi / 4} c_{4}+\frac{\sqrt{2}}{2} c_{5} s_{6+\pi / 4} s_{4}-\frac{\sqrt{2}}{2} s_{5} s_{6+\pi / 4} s_{4} \\
& r_{12}=s_{6+\pi / 4} c_{4}+\frac{\sqrt{2}}{2} c_{5} c_{6+\pi / 4} s_{4}-\frac{\sqrt{2}}{2} s_{5} c_{6+\pi / 4} s_{4} \\
& r_{13}=\frac{\sqrt{2}}{2} s_{4}\left(s_{5}+c_{5}\right) ; r_{21}=-\frac{\sqrt{2}}{2}\left(s_{5}+c_{5}\right) s_{6+\pi / 4}
\end{aligned}
$$




$$
\begin{aligned}
& r_{22}=-\frac{\sqrt{2}}{2}\left(s_{5}+c_{5}\right) c_{6+\pi / 4} ; r_{23}=\frac{\sqrt{2}}{2}\left(-s_{5}+c_{5}\right) \\
& r_{31}=c_{6+\pi / 4} s_{4}+\frac{\sqrt{2}}{2} s_{6+\pi / 4} c_{5} c_{4}-\frac{\sqrt{2}}{2} s_{6+\pi / 4} s_{5} c_{4} \\
& r_{32}=-s_{6+\pi / 4} s_{4}+\frac{\sqrt{2}}{2} c_{6+\pi / 4} c_{5} c_{4}-\frac{\sqrt{2}}{2} c_{6+\pi / 4} s_{5} c_{4} \\
& r_{33}=\frac{\sqrt{2}}{2} c_{4}\left(s_{5}+c_{5}\right)
\end{aligned}
$$

These values for the left leg equation (11) are:

$$
\begin{aligned}
& l_{11}=-c_{6+\pi / 4} c_{4}+\frac{\sqrt{2}}{2} c_{5} s_{6+\pi / 4} s_{4}+\frac{\sqrt{2}}{2} s_{5} s_{6+\pi / 4} s_{4} \\
& l_{12}=s_{6+\pi / 4} c_{4}+\frac{\sqrt{2}}{2} c_{5} c_{6+\pi / 4} s_{4}+\frac{\sqrt{2}}{2} s_{5} c_{6+\pi / 4} s_{4} \\
& l_{13}=-\frac{\sqrt{2}}{2} s_{4}\left(-s_{5}+c_{5}\right) \\
& l_{21}=\frac{\sqrt{2}}{2}\left(-s_{5}+c_{5}\right) s_{6+\pi / 4} \\
& l_{22}=\frac{\sqrt{2}}{2}\left(-s_{5}+c_{5}\right) c_{6} ; l_{23}=\frac{\sqrt{2}}{2}\left(s_{5}+c_{5}\right) \\
& l_{31}=c_{6+\pi / 4} s_{4}+\frac{\sqrt{2}}{2} s_{6+\pi / 4} c_{5} c_{4}+\frac{\sqrt{2}}{2} s_{6+\pi / 4} s_{5} c_{4} \\
& l_{32}=-s_{6+\pi / 4} s_{4}+\frac{\sqrt{2}}{2} c_{6+\pi / 4} c_{5} c_{4}+\frac{\sqrt{2}}{2} c_{6+\pi / 4} s_{5} c_{4}
\end{aligned}
$$

$l_{33}=-\frac{\sqrt{2}}{2} c_{4}\left(-s_{5}+c_{5}\right)$

Solution of (10) and (11) results in the closed formulations:

$\left(\theta_{4}\right)_{R}=\tan ^{-1}\left(\frac{r_{13}}{r_{33}}\right) ;\left(\theta_{4}\right)_{L}=\tan ^{-1}\left(\frac{l_{13}}{l_{33}}\right)$

$\left(\theta_{5}\right)_{R}=\frac{\pi}{4}-\tan ^{-1}\left(\frac{\sqrt{2} r_{23}}{\sqrt{2-2 r_{23}^{2}}}\right)$

$\left(\theta_{5}\right)_{L}=-\frac{\pi}{4}+\tan ^{-1}\left(\frac{\sqrt{2} l_{23}}{\sqrt{2-2 l_{23}^{2}}}\right)$

$\left(\theta_{6}\right)_{R}=\tan ^{-1}\left(\frac{r_{21}}{r_{22}}\right)+\frac{3 \pi}{4}$

$\left(\theta_{6}\right)_{L}=\tan ^{-1}\left(\frac{l_{21}}{l_{22}}\right)-\frac{\pi}{4}$ 